\title{
Agency in Mid-air Interfaces
}

\author{
Patricia Cornelio Martinez ${ }^{1}$, Silvana De Pirro ${ }^{2}$, Chi Thanh $\mathrm{Vi}^{3}$, Sriram Subramanian ${ }^{1}$ \\ ${ }^{1}$ INTERACT LAB, ${ }^{2}$ SARIC, ${ }^{3}$ SCHI LAB \\ University of Sussex, UK \\ [P.Cornelio-Martinez, S.DePirro, C.Vi, Sriram]@ sussex.ac.uk
}

\begin{abstract}
Touchless interfaces allow users to view, control and manipulate digital content without physically touching an interface. They are being explored in a wide range of application scenarios from medical surgery to car dashboard controllers. One aspect of touchless interaction that has not been explored to date is the Sense of Agency (SoA). The SoA refers to the subjective experience of voluntary control over actions in the external world. In this paper, we investigated the SoA in touchless systems using the intentional binding paradigm. We first compare touchless systems with physical interactions and then augmented different types of haptic feedback to explore how different outcome modalities influence users' SoA. From our experiments, we demonstrated that an intentional binding effect is observed in both physical and touchless interactions with no statistical difference. Additionally, we found that haptic and auditory feedback help to increase SoA compared with visual feedback in touchless interfaces. We discuss these findings and identify design opportunities that take agency into consideration.
\end{abstract}

\section{Author Keywords}

Touchless interfaces; haptics; the sense of agency; intentional binding; gestures.

\section{ACM Classification Keywords}

H.5.2 User Interfaces: Theory and methods.

\section{INTRODUCTION}

Recent advances in gesture recognition technologies [31, 40] are driving a new class of interactive systems where a user is able to view, control and manipulate digital content without touching the interface. For example, touchless interactions are being explored as part of medical surgery [51], to design games that benefit children with Autism Spectrum Disorder (ASD) [4], and touchless controllers for car dashboards [2](see Figure 1). There is a strong user appetite for such systems as they are intuitive and enable greater freedom of user-movements.

Permission to make digital or hard copies of all or part of this work for personal or classroom use is granted without fee provided that copies are not made or distributed for profit or commercial advantage and that copies bear this notice and the full citation on the first page. Copyrights for components of this work owned by others than ACM must be honored. Abstracting with credit is permitted. To copy otherwise, or republish, to post on servers or to redistribute to lists, requires prior specific permission and/or a fee. Request permissions from permissions@acm.org.

CHI 2017, May 06-11, 2017, Denver, CO, USA

(C) 2017 ACM. ISBN 978-1-4503-4655-9/17/05 ...\$15.00

DOI: http://dx.doi.org/10.1145/3025453.3025457
One aspect of touchless interaction that has not been studied is the SoA in such interactions. The SoA can be defined as the feeling of one's voluntary actions causing events in the external world [22] and having the awareness of owning the actions" outcomes. This "Attribution of judgement" allows us to distinguish our actions and their sensory effects from those of other people [25].

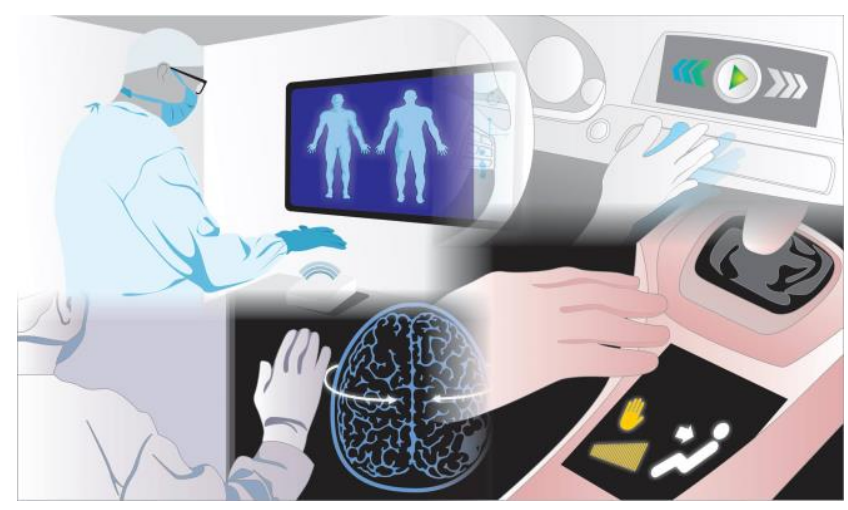

Figure 1. A mosaic of touchless interactions in surgery and driving scenarios.

For example, in touchless applications scenarios in which perceiving a responsive system is relevant (e.g. surgery and driving), if users do not experience perception of causation (causal relationship between action and outcome), they could diminish self-attribution of an unfavorable outcome. However, although this perception is independent of correct performance of the device or system (i.e. personal agency), different interaction paradigms can improve this perception in order to enhance user's SoA through a responsive touchless system. Here, we explored these possibilities

To understand users' SoA when interacting with touchless interfaces, we conducted two user studies. Our studies use the intentional binding paradigm, which provides an implicit and quantitative measure of the SoA [23, 43]. In the first experiment, we compared a camera-based button-click gesture with a physical button press, using both visual and auditory feedback. Our results show that both physical and gestural input modalities produce intentional binding. Additionally, we found that participants exhibited significantly more SoA when the touchless input action was accompanied by an auditory outcome rather than a visual outcome. In the second study, we compared the camerabased click gesture both with and without tactile stimuli to examine if haptic feedback can enhance SoA. Our results show that haptic feedback provides higher intentional 
binding when gesture-based action precedes it, compared with visual feedback.

The main contributions of our paper are:

- We investigate agency effects for touchless gesturebased interaction.

- We demonstrate by implicit and quantitative metrics that touchless gesture-based input modality could be as responsive as a physical touch-based input modality.

- We show that auditory and haptic feedback help to increase user's SoA in touchless interaction compared with visual feedback.

- We discuss our findings and identify design opportunities that take agency into consideration.

\section{RELATED WORK}

The background for our work comes from the areas of experimental paradigms of agency in neuroscience, agency experiments within HCI and touchless interaction systems. Next, we will provide an overview of these areas.

\section{The Sense of Agency}

There is growing interest in investigating an important aspect of self-consciousness that concerns the awareness of being in control of our own actions: the Sense of Agency (SoA), which refers to "Attribution of judgement". Georgieff and Jeannerod defined this phenomenon as a "Who" system that permits the identification of the agent of an action and thus differentiates the self from external agents [18]. This concept has been studied extensively in cognitive neuroscience to analyze how the deficit in people's sense of agency is a consequence of some diseases or mental disorders like schizophrenia; patients with this disorder do not feel they are in control of their own actions and sometimes their thoughts [39].

Currently, two models explain the origins of agency: prediction and postdiction [59]. According to the predictive model, the SoA is generated by the intention to act, which arises from neural processes that regulate initiation of voluntary motor movement [42]. The postdictive model relies on retrospective reflection, so that the SoA arises after perceiving the action's outcome [34, 62]. Here, the perception of causation (causal relationship between action and outcome) is a result of post-action information.

One example of supportive evidence of the predictive model is the work of Benjamin Libet [30], who studied the timeline regarding brain neural activity and the conscious experience of executing a motor movement. His results suggest that the intention of movement is generated by a brain process over which we have no control, as at that moment we are not consciously aware. However, the subjective experience of free will emerges $200 \mathrm{~ms}$ before the actual motor movement. Some researchers have suggested that free will could be described as "free won't" as this process seems to have more to do with the decision to execute an action or not, before the action itself [52].

On the other hand, many studies have shown evidence of the postdictive explanation of agency. Johansson et al. [26] observed postdictive influence over subjects' actions based on choice blindness. In this study, participants were asked to visually choose one option among others. Then, the experimenters swapped the participants' chosen option with a new one, and presented this new option as their original choice. When participants were asked to explain the reason for their choice, they tried to justify why they chose the swapped option, even though it was clearly different to the original choice.

Another example is the study by Takahata et al. [60] who conducted an experiment where participants were primed with rewarding and punishing outcomes by associating auditory stimuli with positive, neutral and negative monetary outcomes. Their results showed that participants attributed an action to themselves depending on outcome condition; they generally attributed the action to an external factor when its effect produced a negative outcome.

Body ownership also plays an important role in the experience of agency. Participants in [3] falsely attributed an action (speaking) to themselves. The experiment consisted of a virtual reality scene in which participants saw a life-size speaking avatar seen from the first person perspective through a virtual mirror. Participants received thyroid cartilage vibrotactile stimulation synchronized with the avatar's speech. The movements of the virtual body and participant body were also synchronized so that they created the illusion of body ownership.

They demonstrated that participants thought they were speaking the words when they actually were not. In a more recent work they found illusion of agency over walking in seated participants [28]. These findings suggest that people experience SoA even when there is no previous intention to act i.e. in absence of prediction, priming or cause preceding the effect. Although the studies differ in explanations about the initiation of SoA, both models are considered valid [59].

\section{Agency in $\mathbf{H C l}$}

Although the experience of agency is central in cognitive neuroscience, recent research has focused on studying how personal agency changes with use of technology. These studies have opened a new area which aims to explore how "in-control" users feel when interacting with an interface, i.e. have the awareness to say, "I am, who is controlling this". McEneaney et al. [37] executed a series of experiments to demonstrate that the experience of agency not only applies for physical situations but also in HCI. They focused on answering: "Are agency effects observed in desktop computing environments typical of HCI?"

They based their studies on measuring perception of clickresponses through visual stimuli on-screen and auditory feedback to compare human-initiated actions with 
computer-controlled actions. Their results showed that an agency effect exists in typical HCI desktop computer environments. This finding supports the claim that user perception of on-screen events depends on agency cues. However, they found that the perception in time of participants differed depending on whether an auditory effect followed a machine or human-initiated click action. Coyle et al. [12] compared a new input modality (Skinput [24]) with physical interaction (button-press) to explore the experience of agency in HCI environments. Skin-based input modality consisted of a piezoelectric microphone on participants' forearm so that a tap on the skin can be recognized as a "button-press" action, preceding an audio feedback in response. The results showed that skin-based input could elicit greater SoA unlike typical keyboard input.

In another example, Limerick et al. [32] explored voice command input. This technique consists of asking participants to say the word "go" as an instruction/action preceding an audio feedback. Their results showed a low SoA in this input modality, suggesting that this low feeling of control contributes to the low uptake of speech interfaces for interactive applications, despite the availability of high accuracy voice recognition techniques (e.g. $97.3 \%$ recognition rate). This research suggests that a system that evokes a low sense of agency will discourage users from using it, preventing widespread use of the system. On the other hand, the research of Coyle creates a large opportunity for on-body interaction systems. We need a similar understanding of the SoA for touchless systems in order to improve touchless interface design and thus enable wider uptake of such systems.

\section{Touchless Systems}

Interactive systems that use a touchless approach typically require no physical contact with a surface or object, avoiding the constraints of ordinary interaction paradigms (e.g. mouse and keyboard). These systems often rely on gesture-tracking technologies to detect mid-air gestures. The most common approaches rely on optical technology [61, 68] and electromyography (EMG) [38, 50]. However more recent devices offer higher resolution of gesturesensing based on radar [31] and sonar [49] technologies.

Taking advantage of its properties, touchless systems are being deployed to perform interactions in many critical situations such as surgery and dashboard control. Touchless manipulation of medical images allows surgeons to maintain the sterile environment required in surgery, without the help of assistants [51]. Another example is driving; today there are many dashboard panels that allow users to control car elements from a distance [2, 29]. The use of gesture recognition and proximity to manipulate car controllers allows the user to release the visual channel, and thus aims to promote a safer driving environment.

Although mid-air gesture-based devices may consist of a wide range of capabilities, most radar, sonar or optical tracking-based gestures typically share common characteristics with mice and tablets. In both devices the main interplay consists of pointing and clicking actions [65]. In these mid-air gesture interaction systems, pointing is represented by hand tracking and clicking is represented by "activation gestures" [65], which define the intention to communicate with the system [20]. These gestures must be natural and intuitive, but uncommon, so that they are not performed accidentally [7]. Following this, the user expects a confirmation of the activation, i.e. a perceptible response from the system. This refers to "system attention" [5], which is attained with multisensory feedback. Feedback is important in touchless systems as there is no physical contact with an object (e.g. floating images or virtual keyboard). However, it is not necessary to physically touch an object to have the perception of a "button-press" if it is associated with an effect in response.

\section{Visual, audio and haptic feedback}

Touchless interaction can be helped by sensory effects in order for the user to perceive "system attention" [5]. This can be achieved by providing users with multisensorial feedback, i.e. visual, auditory and haptic [20]. Freeman and Lantz added light, audio and tactile displays to assist users to know "where to gesture" [16]. Markussen et al. implemented a gestural typing system helped with visual feedback through a virtual keyboard [36]. Liu et al. added visual hand-cursors on-screen to make users know the state of the bare-hand postures and gestures [33]. Wu and Rank explored different audio feedback designs for hand gestures for encouraging immersion in games [66]. In a recent work they found that in-air gestures with responsive audio feedback leads to a higher immersion and enjoyment in video games [67]. Müller et al. developed a technique to "touch" and manipulate sound in mid-air by combining audio, visual and tactile feedback [48].

A common criticism of touchless systems is that users lack haptic feedback for action confirmation. However, mid-air haptic feedback is a recent technique to make the user aware of "system attention" in touchless interaction. Airwave [21], UltraHaptics [8] and AIREAL [57] are examples of emerging systems that can provide this missing tactile feedback in mid-air with bare hands. This technology allows users to perceive tactile sensation even in the absence of physical objects. Based on this approach, Monnai et al. proposed a system to interact with floating images, using not only visual feedback (through light beams), but also mid-air haptic feedback through ultrasound in order to create the sensation of touching a virtual screen [41]. In a more recent work, Makino et al. introduced a system to clone real objects into virtual ones. It consisted of floating images that replicate haptic properties of real objects using ultrasound, providing realistic interaction of touch in mid-air without wearable devices [35].

The above examples represent complex systems of touchless input commands with different kinds of feedback. 
However, the role of agency during the interaction with these systems has not been investigated. In other words, it is unclear if adding tactile feedback helps user feel SoA when interacting with touchless systems. We believe that agency implication should be considered in touchless interface design in order to improve user involvement, intuitiveness and instinctive sense of control during the interaction.

This is the seventh of Shneiderman's Eight Golden Rules of Interface Design; this rule indicates that interface design should "support an internal locus of control"[56] which refers to users' need to feel they are the agents of the system's responses (i.e. "they are in charge of the system"). This is a relevant aspect for new application scenarios (e.g. surgery and driving) in which feeling ownership of the outcomes of one's actions is essential. To investigate user's SoA beyond traditional input in these new scenarios, we explored agency by employing the intentional binding paradigm and the Libet clock in a set of input command actions (physical and touchless) and sensory responses that include audio, visual and haptic feedback.

\section{THE INTENTIONAL BINDING PARADIGM}

We used the intentional binding paradigm for our studies. It was developed to provide an implicit and quantitative measure of the SoA [23]. In 2002 Patrick Haggard showed that the perceived time of a voluntary action and its sensory outcomes are shifted towards each other, so that the interval between action and outcome is perceived as shorter than it actually is, leading to a perception of compression of time [23] (see Figure 2 right). As shown in Figure 2 right, the action binding represents the interval between the actual and perceived action; it occurs when the action is perceived to occur later than the moment when it actually did. Similarly, the outcome binding represents the interval between the actual and perceived outcome, it occurs when the sensory effect is perceived earlier than the moment when it actually happened. The sum of these two elements represents a total binding value. Consequently, higher total binding value is related to a higher SoA [12, 14, 45].

According to this method, the action binding and outcome binding can be measured quantitatively. They are calculated from four conditions (see Table 1) consisting of two baseline- (baseline action \& baseline outcome) and two active- (active action \& active outcome) conditions. As illustrated in Table 1, in the action baseline condition, participants performed the action (physical or touchless) but receive no feedback. In the outcome baseline condition, participants received feedback (visual, auditory or haptic) without performing any action. In the active conditions, both action and outcome occurred. During the task, both actual time (the time logged by the system) and perceived time (reported by the user using a Libet clock) of the action and outcome was recorded. The errors were calculated by the difference between perceived and actual moments of time. Following this, the intentional binding is calculated through the formulas shown in Figure 3 [46].

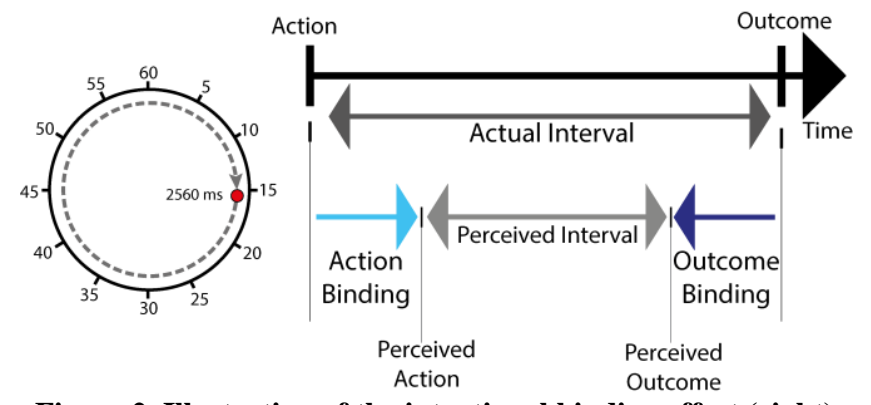

Figure 2. Illustration of the intentional binding effect (right). The Libet clock (left).

\begin{tabular}{|c|c|c|c|c|}
\hline \multicolumn{5}{|c|}{ Measurement Blocks } \\
\hline Condition & Action & Outcome & \begin{tabular}{|c|} 
Participant \\
Report
\end{tabular} & Error \\
\hline $\begin{array}{c}\text { Baseline } \\
\text { action }\end{array}$ & dim & None & Action & Error $=$ perceived time - actual time \\
\hline $\begin{array}{l}\text { Baseline } \\
\text { outcome }\end{array}$ & None & $(\forall))$ & Outcome & Error $=$ perceived time - actual time \\
\hline $\begin{array}{l}\text { Active } \\
\text { action }\end{array}$ & $\mathrm{dm}$ & D)) & Action & Error $=$ perceived time - actual time \\
\hline $\begin{array}{c}\text { Active } \\
\text { outcome }\end{array}$ & ftr & Ð)) & Outcome & Error $=$ perceived time - actual time \\
\hline
\end{tabular}

Table 1. Intentional binding measurement blocks and calculations for error estimation.

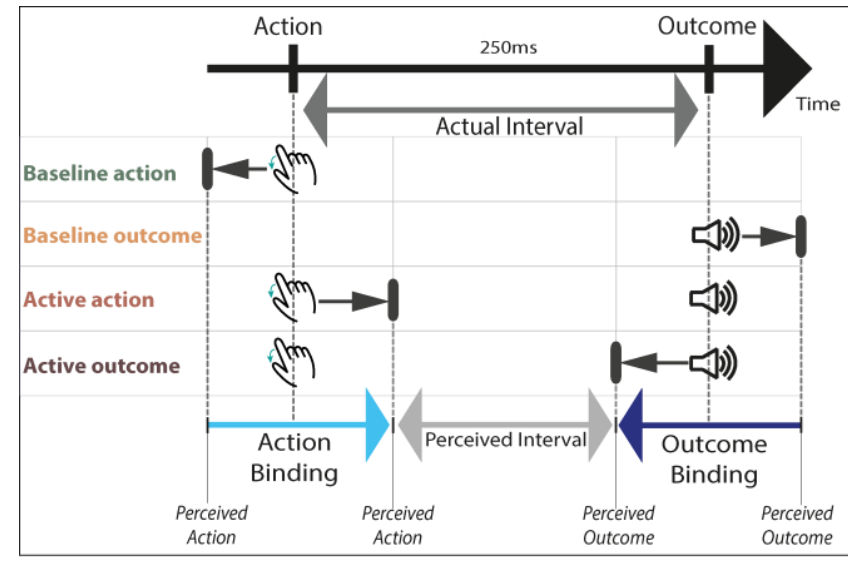

Error $=$ Perceived time - Actual time

Action Binding $=$ Active action error - Baseline action error

Outcome Binding = Active outcome error - Baseline outcome error Total Binding $=$ Action Binding - Outcome Binding

Figure 3. Intentional binding conditions and calculation formulas. For action, participants executed either a physical button-press or a touchless button-click gesture. For outcome, they received one action confirmation: visual on-screen, auditory (a beep), vibrotactile or mid-air haptic feedback.

As shown in Figure 3, in baseline conditions participants perceived the action earlier but the outcome later when compared to the actual time. However, in active conditions the effect was the opposite. The active conditions create an effect where action and outcome are shifted towards each other [23]. This is evidence of both predictive and postdictive influence of the origination of agency in the intentional binding paradigm $[13,43]$. 


\section{The Libet clock}

We employed Libet's method to measure participants' time-subjective experience, in order to investigate volitional control of movement and thus to record perception of time (the Libet clock). It consists of a clock that rotates clockwise once every 2560ms (see Figure 2 left). Participants reported the position of the clock at the moment when they either performed the action or received the outcome (see Table 1) to indicate their perceived time. The experience of agency can be also measured with alternative methods, for example "Interval estimation" [15, 43], which consists of reporting an estimate of the time interval between the action and the outcome. Another method is self-reporting questionnaires and scales that are related to binary answers about whether the user was the agent of the action or not. However, the intentional binding paradigm with the Libet Clock has been shown as a robust technique to implicitly measure the SoA [43, 45, 53, 54]. We therefore use this method in our experiments.

\section{TOUCHLESS BUTTON CLICK}

In order to investigate the relationship between states of the in-air gesture input and the system's responses, we wanted to explore how gesture actions influence agency. We measured intentional binding during simple microinteractions typical of desktop computing environments. We based our selection gesture on a study by Saffer, who states that "The best, most natural designs, then, are those that match the behavior of the system to the gesture humans might actually do to enable that behavior. Simple examples include pushing a button to turn something on or off" [55]. Consequently, we chose a fundamental gesture action to use (touchless button-click) in order to compare it with typical touch input (button-press).

In this context, a button-press movement is common in our everyday interaction with computers and smartphones. Besides, it can be reliably tracked with devices such as Leap Motion, which is specifically focused on hand and fingers tracking. In common desktop computing environments, a physical button-press generally produces three kinds of effect: (1) visual on-screen: when we press a button or key of the keyboard we normally expect a visual change on-screen (e.g. typing tasks), (2) auditory feedback: because we can perceive a click sound through mechanical pressure on the actuator; and (3) haptic: because of the obvious physical contact with the mechanoreceptors of the skin. Therefore, we provided participants with visual, auditory and haptic feedback as the outcome of our physical and gestural action input to examine how states of input (physical and touchless) map onto states of the system.

\section{INVESTIGATING AGENCY IN TOUCHLESS INTERFACES}

Touchless systems are being used in a wide range of applications; however, the role of SoA in this kind of interaction is unknown. Does the user perceive a touchless command as being as responsive as a physical one? Does haptic feedback help to increase user's SoA in touchless interfaces? To answer these questions, we conducted two studies. In our Study 1, we explored touchless input modality and compared it with physical-based input. So far, only auditory and visual outcomes have been employed as a means of action confirmation to investigate agency (as mentioned in related work section); however, in our Study 2, we introduced haptic feedback (vibrotactile and mid-air) as a new output modality to investigate if tactile sensation can enhance users' SoA in touchless interactions.

\section{Method and Materials}

Participants judged their perception of time by reporting the position of a rotating dot around a Libet clock at the moment when they either executed an action (baseline action and active action blocks) or received the feedback (baseline outcome and active outcome blocks) as shown in Table 1. The numbers of the clock were not used in order to avoid creating visual patterns during the task. This is because in pilot studies we noticed that participants tended to "identify" with their gaze a number as a reference, (e.g. "I'm going to do the action when the dot reaches the number 3"). This does not reflect the volition/urge to execute a motor action. Thereby participants used an external controller (Griffin Powermate USB Controller) to place the dot on the perceived position. The Libet clock size 500 pixels in diameter, was placed at the center of a screen (24 inch, $1920 \times 1080$ resolution). The perceived and actual times were recorded to calculate the intentional binding. In the trials with user-performed action, the action was either a touchless click gesture or a physical button-press. The outcome was presented in one of four different feedback methods: on-screen visual, auditory (a beep), wearable vibrotactile, and mid-air haptic feedback.

\section{Gesture Action}

Participants moved their index finger, mimicking a pressbutton action (i.e. up-down finger movement of $2 \mathrm{~cm}$ ). The gesture was captured using a Leap Motion controller with capture rates of about $300 \mathrm{fps}$. Participants rested their hand (palm down) at a fixed position of about $20 \mathrm{~cm}$ height from the surface of the Leap Motion device in all feedback conditions preceding the gesture (see Figure 4). After a period of $250 \mathrm{~ms}$ a sensory effect was given to participants.

\section{Auditory Outcome}

Auditory stimulus is the common sensory effect used in the intentional binding paradigm. We considered audio feedback to have baseline comparison with new outcome modalities. In the conditions when there was auditory feedback, participants heard a tone that lasted $200 \mathrm{~ms}$ at $900 \mathrm{~Hz}$ in frequency using headphones. However, they always wore headphones during the full study.

\section{Visual Outcome}

Visual feedback was in the form of an on-screen button (250 pixels in diameter) that was presented at the center of the screen, and inside the Libet clock. When participants 
performed the click gesture, they could see the animation of this button changing state (the button sank as if it had been pressed; changed from red to green; and returned to its original state after $200 \mathrm{~ms}$ ). The procedure for presenting visual stimuli and the Libet clock is similar to previous studies [37, 47]. Possible time delays due to the refresh rate of the screen used in our study $(60 \mathrm{~Hz})$ in the visual conditions on-screen, including the rotation of the Libet clock, was compensated for by following the procedure of previous studies [58]. We executed a preliminary test with a photodetector and high-speed camera placed in the middle of the screen in order to count the number of frames shown within specific periods of time. This was done in order to identify and compensate for missing frames. Our system was consistent in missing one frame in each trial, so to correct this delay, we subtracted the duration of one tick (16.66ms) from our interval durations as in [17].

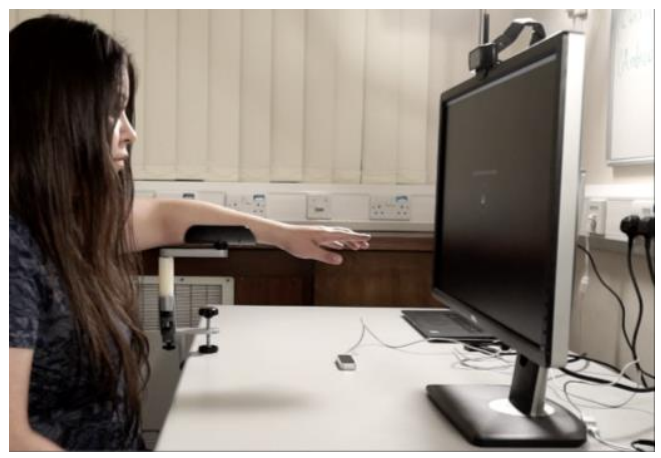

Figure 4. Experimental setup.

\section{Vibrotactile Haptic Outcome}

Vibrotactile feedback was given to participants using a wearable glove with an embedded coin vibration motor (model 310-103 by Precision Microdrives), $1 \mathrm{~cm}$ in diameter and positioned in the glove so that the vibration is provided on a participant's fingertip (index finger). This motor vibrated at a speed of $12,000 \mathrm{rpm}$ and $250 \mathrm{~Hz}$ in frequency. The typical rise time of $87 \mathrm{~ms}$ was compensated for to preserve timing as accurately as possible. Each vibration lasted $200 \mathrm{~ms}$, which was easily recognizable over the tactile channel [19]. Participants did not wear the glove during visual, auditory and mid-air haptic feedback blocks.

\section{Mid-air Haptic Outcome}

Mid-air haptic feedback was provided using the UltraHaptics kit [8]. This device uses low-intensity and low-frequency ultrasound pressure waves to create multiple focal points in mid-air for tactile-sensation. The user can perceive the focal points using bare hands due to the receptors in the hand evoking a haptic sensation. To equalize two haptic feedback conditions in terms of stimulation area, we simulated vibrotactile outcome features with an UltraHaptics kit. Five focal points were created on participants' fingertip (index finger) to cover an area of $1 \mathrm{~cm}^{2}$ with the same frequency as the vibrotactile condition $(250 \mathrm{~Hz})$. The stimulation lasted for $200 \mathrm{~ms}$.

\section{STUDY 1. TOUCHLESS VS PHYSICAL}

In this experiment, we compared physical-based and gestural-based touchless inputs preceding auditory and visual feedback as the outcome. This resulted in four combinations of action + outcome: physical \& auditory, physical \& visual, gestural \& auditory and gestural \& visual, as shown in Figure 5.

\section{Procedure}

Participants were asked to sit in front of a screen at a distance of about $100 \mathrm{~cm}$. Every trial started when they pressed a footswitch to indicate they were ready to start. After this, a Libet clock with a rotating dot was presented at the center of a screen. The dot always started at a random position. After one full revolution of the dot, participants were asked to perform the action: a physical button-press using a keyboard (space key) or a click gesture in mid-air. For touchless action, the hand always stayed palm down and rested on top of a supporting structure (as in Figure 4). For physical action, this structure was not used and the Leap Motion device was replaced by a computer keyboard. Participants always executed the action (gestural and physical) using their dominant hand.

After a period of $250 \mathrm{~ms}$, the outcome was presented in the form of auditory (a beep) and visual feedback on-screen. Then, participants judged their perception of time by reporting the position of the dot on the clock. Participants wore noise-cancelling headphones to eliminate any audible noise from the devices. Participants performed 20 trials in each condition resulting in 320 trials per participant $(20$ trails $\mathrm{x} 4$ intentional binding blocks $\mathrm{x} 4$ combinations of action + feedback). The experiment was completed in a maximum time of $90 \mathrm{~min}$; there was a short break between conditions. Figure 5 shows the procedure of a single trial.

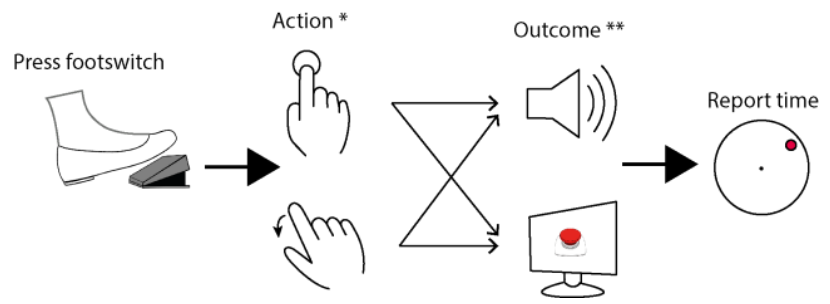

Figure 5. Experimental trial of Study 1 (*not done in baseline outcome blocks, ** not done in baseline action blocks).

\section{Participants}

Twelve right-handed participants (4 Male, mean age $=30.92$ years, $\mathrm{SD}=3.03$ ) took part in the experiment. They had normal or corrected-to-normal vision. The local ethics committee approved this study and participants were not paid for their participation.

\section{Results}

A Repeated Measure design was used to compare the effects of touchless input modality with physical-based input and visual and auditory feedback. We report the 

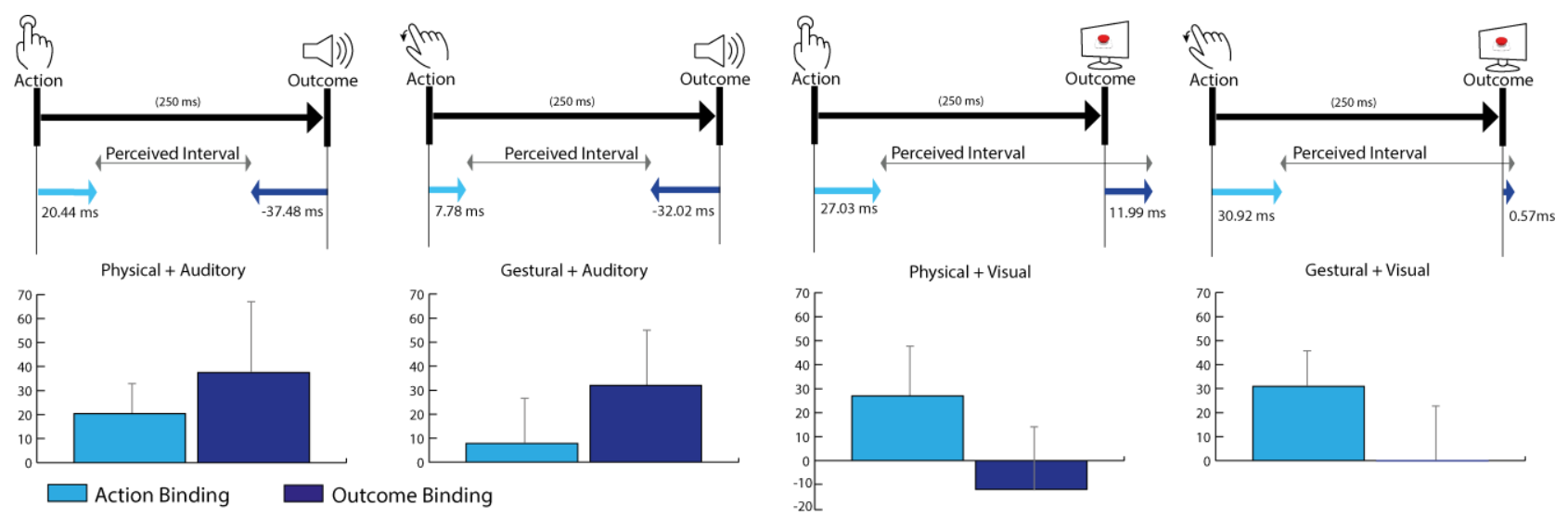

Figure 6. Average of action binding and outcome binding in milliseconds of each action and outcome modality. The sign of outcome binding effects on the chart bars has been inverted to allow for comparison with action binding. Error bars represent standard error of mean.

partial eta squared $\left(\eta_{\mathrm{p}}^{2}\right)$ as a measure of effect size. According to Cohen [11], a value of 0.01 is considered a small effect, 0.06 a medium effect and a value of 0.14 or greater, a large effect size.

\begin{tabular}{|c|c|c|c|}
\hline $\begin{array}{c}\text { Action } \\
\text { Feedback }\end{array}$ & Action Binding & Outcome Binding & Total Binding \\
\hline $\begin{array}{l}\text { Physical } \\
\text { Auditory }\end{array}$ & $\begin{array}{c}20.44 \mathrm{~ms} \\
(43.79 \mathrm{~ms})\end{array}$ & $\begin{array}{c}-37.48 \mathrm{~ms} \\
(106.23 \mathrm{~ms})\end{array}$ & $\begin{array}{c}57.92 \mathrm{~ms} \\
(103.88 \mathrm{~ms})\end{array}$ \\
\hline $\begin{array}{c}\text { Physical } \\
\text { Visual }\end{array}$ & $\begin{array}{c}27.03 \mathrm{~ms} \\
(73.50 \mathrm{~ms})\end{array}$ & $\begin{array}{c}11.99 \mathrm{~ms} \\
(92.28 \mathrm{~ms})\end{array}$ & $\begin{array}{c}15.04 \mathrm{~ms} \\
(109.42 \mathrm{~ms})\end{array}$ \\
\hline $\begin{array}{l}\text { Gestural } \\
+ \\
\text { Auditory }\end{array}$ & $\begin{array}{c}7.78 \mathrm{~ms} \\
(66.81 \mathrm{~ms})\end{array}$ & $\begin{array}{l}-32.02 \mathrm{~ms} \\
(81.73 \mathrm{~ms})\end{array}$ & $\begin{array}{c}39.80 \mathrm{~ms} \\
(106.01 \mathrm{~ms})\end{array}$ \\
\hline $\begin{array}{c}\text { Gestural } \\
\text { Visual }\end{array}$ & $\begin{array}{c}30.92 \mathrm{~ms} \\
(52.76 \mathrm{~ms})\end{array}$ & $\begin{array}{c}0.57 \mathrm{~ms} \\
(81.25 \mathrm{~ms})\end{array}$ & $\begin{array}{c}30.87 \mathrm{~ms} \\
(68.30 \mathrm{~ms})\end{array}$ \\
\hline
\end{tabular}

Table 2. Average of action, outcome and total binding in milliseconds (with standard deviation in brackets) grouped by combination of action \& outcome.

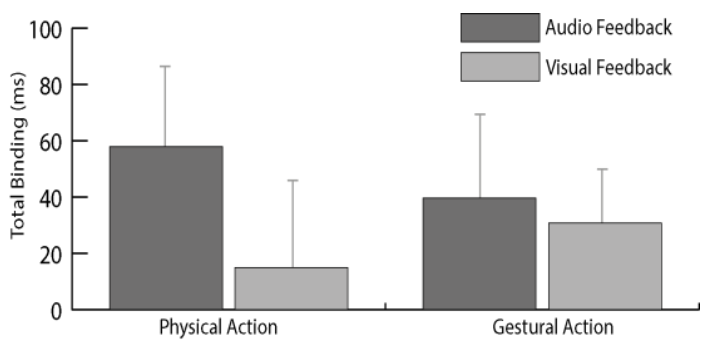

Figure 7. Average of total binding in milliseconds for each combination of action and outcome. Error bars represent standard error of mean.

A 2X2 within subjects' ANOVA, with the type of action (touchless gesture-based click vs physical button-press) and the type of feedback (visual vs auditory) as factors, revealed no significant effect of type of action on total binding $F_{(1,11)}=0.003, \quad p=0.96, \quad \eta_{\mathrm{p}}{ }^{2}=0.00$. We also found no significant interaction between the type of action and type of feedback $F_{(1,11)}=0.63, p=0.45, \eta_{\mathrm{p}}{ }^{2}=0.05$. However there was a significant main effect of the type of feedback $F_{(1,11)}=5.31, p=0.04, \eta_{\mathrm{p}}{ }^{2}=0.33$ with the auditory feedback scoring higher compared to the visual feedback. Figure 7 shows the average total binding with different action and feedback modalities.

An identical ANOVA was then performed for the action binding, showing no significant interaction $F_{(1,11)}=0.36$, $p=0.56, \eta_{\mathrm{p}}{ }^{2}=0.03$, and no main effect of the type of action $F_{(1,11)}=0.12, p=0.74, \eta_{\mathrm{p}}{ }^{2}=0.01$ and the type of feedback, $F_{(1,11)}=0.79, p=0.39, \eta_{\mathrm{p}}{ }^{2}=0.07$.

The outcome binding, however, showed a significant main effect of the type of feedback $F_{(1,11)}=9.17, \mathrm{p}=0.01$, $\eta_{\mathrm{p}}{ }^{2}=0.45$, with auditory outcome producing an increased binding in both the physical button-press ( $M=-37.48 \mathrm{~ms}$, $S D=106.23 \mathrm{~ms}$ ) and the touchless gesture-based click ( $M=-32.02 \mathrm{~ms}, S D=81.73 \mathrm{~ms}$ ) compared to visual feedback respectively in the physical action $(M=11.99 \mathrm{~ms}$, $S D=92.28 \mathrm{~ms}$ ) and in the touchless gesture-based click $(M=0.57 \mathrm{~ms}, S D=81.25 \mathrm{~ms})$. A breakdown of these means in relation to action and outcome binding is shown in Table 2. Figure 6 shows action binding and outcome binding effects.

\section{Discussion of Study 1}

Our results from the Study 1 revealed an intentional binding effect when both input modalities gestural and physical preceded an auditory feedback. However, this effect was not observed with visual feedback. As shown in Figure 6 the visual outcome did not shift towards the action. This suggests that the touchless system exhibited significantly more intentional binding when the input action was accompanied by auditory outcome compared with visual outcome. As expected, the physical button-press preceding an auditory outcome produced intentional binding, as shown in a large number of studies on SoA.

Interestingly we found no statistically significant difference in the action binding across the different combinations of action and outcome. This could suggest that participants may have perceived the touchless action to be as responsive as the physical action in terms of intentional binding, even when the touchless action did not involve typical characteristics of touching and object i.e. proprioceptive perception. The proprioceptive perception plays an 
important role in terms of feeling an immediate haptic feedback (as in pressing a physical button) additionally from the feedback system. In the previous work from Coyle et al. [12], participants reported increased intentional binding for skin-based input modality as this action involved tactile sensation in both the finger and the arm. Thereby, this seems a challenge for touchless action where implicit tactile feedback is not committed.

However, although in our touchless condition there was not a simultaneous action-feedback like in physical buttonpress, interestingly we still found an intentional binding effect, as the touchless action execution always involved participants' motor movement followed a prior intention. Previous studies have suggested that the SoA principally arises due to internal motor signals $[6,42]$ and also that intention to act influences action attribution, when reafferent signals (e.g. motor or visual) match with intention retrospectively [9, 10, 64]. Thereby ideomotor signals produced by the touchless action could have served as a contributory factor in our results on intentional binding.

Furthermore, we also attribute these findings to the influence of the postdictive model of origination of agency. As we state, "it is not necessary to physically touch an object to have the perception of a "button-press" if it is associated with an effect in response (see Visual, audio and haptic feedback section). Although the touchless action did not involve immediate tactile feedback, participants always received a confirmation with a visual or auditory outcome. Similar accounts were reported in [3, 28, 63], where subjects reported feelings of agency even when there was no cause preceding the effect, but just the effect itself. Yet in our studies participants always had an intention to act and thereby a motor movement preceding an outcome. This could have contributed to the intentional biding effect shown in our results.

\section{STUDY 2. TOUCHLESS VISUAL \& HAPTICS}

This experiment aimed to investigate if haptic feedback can improve participants' SoA in gesture-based touchless interaction. For this, we measured intentional binding both with and without haptic feedback.

\section{Procedure}

Participants in Study 2 used the same experimental procedure used in Study 1, with one exception. Whereas participants in Study 1 performed two kinds of actions (physical and touchless) and received two kinds of feedback (auditory and visual), in the second study participants performed only the touchless-based action and received visual, vibrotactile and mid-air haptic feedback (Figure 8). Both kinds of haptic feedback were provided on participants' dominant hand (index finger). Participants wore noise-cancelling headphones to eliminate any audible noise from the devices. Participants performed 30 trials for each condition resulting in 360 trails per participant (30 trials $\times 4$ intentional binding blocks $\times 3$ combinations of action + feedback). The experiment was completed in a maximum time of $90 \mathrm{~min}$; there was a short break between conditions. Figure 8 shows the procedure of a single trial.

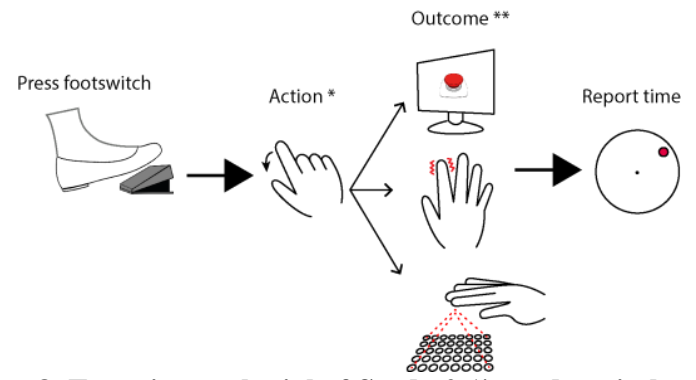

Figure 8. Experimental trial of Study 2 (*not done in baseline outcome blocks, ${ }^{* *}$ not done in baseline action blocks).

\section{Participants}

Twelve right-handed participants (4 Female, mean age $=30.33$ years, $S D=3.86$ ), took part in the experiment. They had normal or correct-to-normal vision. The local ethics committee approved this study and participants were not paid for their participation.

\section{Results}

A One-way Repeated Measure ANOVA was conducted to compare the effect of the three type of feedback (visual vs vibrotactile vs mid-air haptic) on the action, outcome and total binding. Results show a significant effect on the total binding $F_{(2,22)}=4.96, p=0.02, \eta_{p}{ }^{2}=0.31$ depending on the type of feedback. Post-hoc comparisons using Bonferroni correction showed that there is a statistically significant difference in the total binding specifically in the mid-air haptic feedback $(M=84.21 \mathrm{~ms}, S D=111.35 \mathrm{~ms})$ compared to the visual $(M=-6.41 \mathrm{~ms}, S D=82.98 \mathrm{~ms}), p=0.02$; but no such difference was found compared to the vibrotactile condition $(M=40.77 \mathrm{~ms}, \quad S D=89.84 \mathrm{~ms}), \quad p=0.69$. The difference between the visual condition and vibrotactile was also not significant, $p=0.23$. Figure 10 shows the average total binding with different action and feedback modalities.

We found that the action binding was not significantly affected by the type of feedback $F_{(2,22)}=0.27, p=0.76$, $\eta_{p}{ }^{2}=0.02$. However, crucially the outcome binding showed a significant difference $F_{(2,22)}=0.6 .74, \quad p=0.005, \quad \eta_{p}{ }^{2}=0.38$. Post-hoc comparisons using Bonferroni correction showed that the outcome binding was significantly greater in the mid-air haptic condition $(M=-64.79 \mathrm{~ms}, \quad S D=79.58 \mathrm{~ms})$ compared to the visual $(M=12.68 \mathrm{~ms}, \quad S D=66.07 \mathrm{~ms})$ condition $p=0.02$, but there was not statistically significant difference between the mid-air haptic and the vibrotactile feedback $\quad(M=-29.13 \mathrm{~ms}, \quad S D=69.75 \mathrm{~ms}), \quad p=0.69 \mathrm{~ms}$. Additionally, we found no significant difference between the vibrotactile and the visual $p=0.23$.

These findings suggest that mid-air haptic feedback produces a strongest effect in the intentional binding values and specifically in the outcome binding compared to the other modalities. A breakdown of means in relation to 

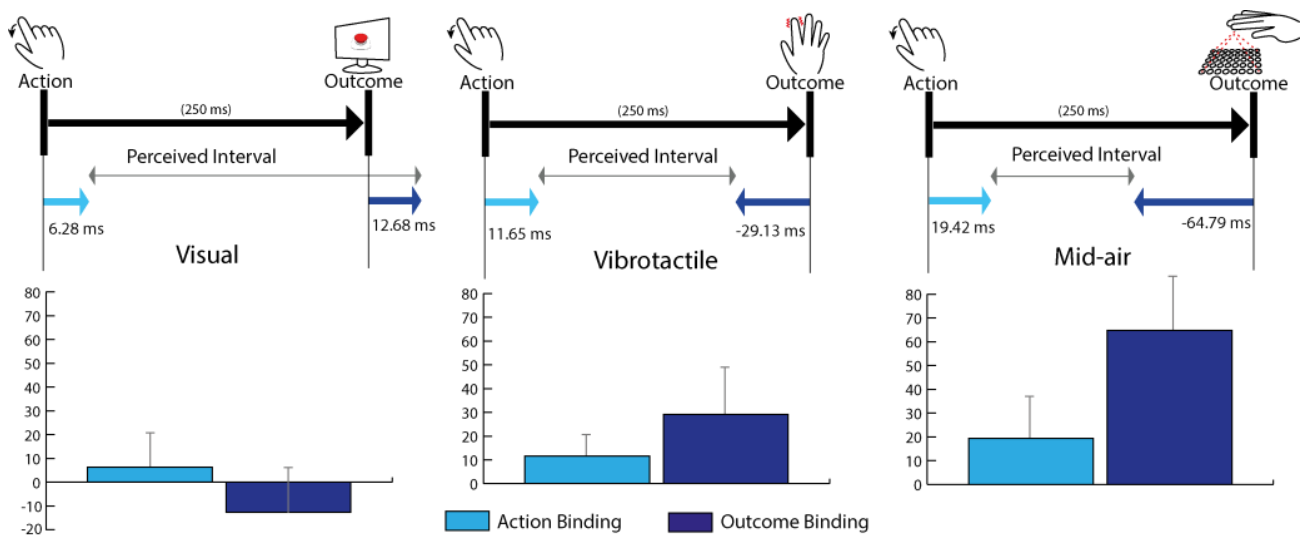

Figure 9. Average of action binding and outcome binding in milliseconds for each feedback type (visual, vibrotactile, and mid-air). The sign of outcome binding effects on the chart bars has been inverted to allow for comparison with action binding. Error bars represent standard error of mean.

action and outcome binding is shown in Table 3. Figure 9 shows action binding and outcome binding effects.

\begin{tabular}{|c|c|c|c|}
\hline Feedback & Action Binding & Outcome Binding & Total Binding \\
\hline Visual & $\begin{array}{c}6.28 \mathrm{~ms} \\
(49.55 \mathrm{~ms})\end{array}$ & $\begin{array}{c}12.68 \mathrm{~ms} \\
(66.07 \mathrm{~ms})\end{array}$ & $\begin{array}{c}-6.41 \mathrm{~ms} \\
(82.98 \mathrm{~ms})\end{array}$ \\
\hline \multirow{2}{*}{ Vibrotactile } & $\begin{array}{c}11.65 \mathrm{~ms} \\
(32.39 \mathrm{~ms})\end{array}$ & $\begin{array}{c}-29.13 \mathrm{~ms} \\
(69.76 \mathrm{~ms})\end{array}$ & $\begin{array}{c}40.77 \mathrm{~ms} \\
(89.84 \mathrm{~ms})\end{array}$ \\
\hline Mid-air & $19.42 \mathrm{~ms}$ & $-64.79 \mathrm{~ms}$ & $84.21 \mathrm{~ms}$ \\
& $(62.22 \mathrm{~ms})$ & $(79.58 \mathrm{~ms})$ & $(111.35 \mathrm{~ms})$ \\
\hline
\end{tabular}

Table 3. Average of action, outcome, and total binding (with standard deviation in brackets) grouped by feedback type.

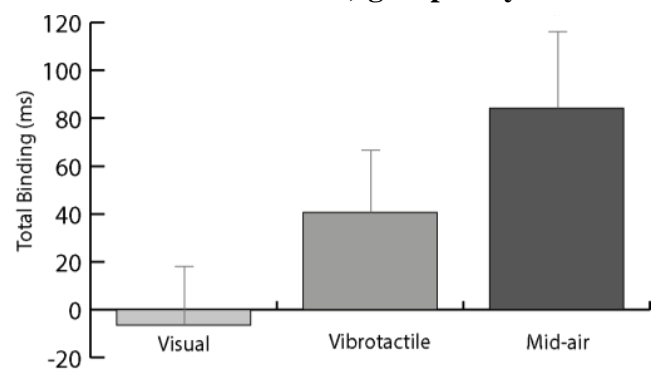

Figure 10. Average of total binding in milliseconds for each feedback type (visual, vibrotactile, and mid-air). Error bars represent standard error of mean.

We additionally performed further analysis using independent sample t-test to compare the effect of the intentional binding with auditory feedback in touchless modality in the Study 1 with the mid-air haptic feedback in the Study 2. Results showed no significant difference on the total binding $t(22)=0.99, p=0.33$ between the auditory condition of the Study $1(M=39.80 \mathrm{~ms}, S D=106.02 \mathrm{~ms})$, and the mid-air haptic condition $(M=84.21 \mathrm{~ms}, S D=111.35 \mathrm{~ms})$ of the Study 2. These results were also not significant for the outcome binding $t(22)=0.68, p=0.32$, in the auditory condition $(M=-32.02 \mathrm{~ms}, S D=81.73 \mathrm{~ms})$ compared to the mid-air haptic condition $(M=-64.79 \mathrm{~ms}, S D=79.58 \mathrm{~ms})$.

\section{Discussion of Study 2}

Our results from the Study 2 revealed an intentional binding effect when the touchless input modality preceded a haptic feedback. However, this effect was not observed with visual feedback similar to Study 1. This suggests that the touchless system exhibited significant higher intentional binding when participants received a haptic confirmation rather than a visual confirmation. Crucially we found no statistically significant difference in action binding values across the outcome modalities.

Both haptic feedback conditions (vibrotactile and mid-air) shifted towards the touchless action. Interestingly we found no statistically significant difference for outcome binding between these two haptic conditions. We set both outcome conditions with the same characteristics as much as possible. This is because vibrotactile feedback is higher in intensity compared with ultrasound. However, by creating five focal points of ultrasound overlapping each other to cover the same area as the vibrotactile stimuli, we could equalize between these two conditions.

\section{GENERAL DISCUSSION}

Our results revealed the existence of intentional binding effect in touchless gesture-based interactive applications. From our two studies, we found that gesture-based system exhibited significant higher intentional binding when the input action was accompanied by haptic or auditory outcomes compared with visual outcome. Our results from Study 1 showed action binding effect in both physical and touchless interactions with no statistically significant difference, possibly suggesting that that our click gesture input could be as responsive as the physical action in terms of intentional binding, even when no simultaneous actionfeedback occurred like in physical touch events. We attribute this result to ideomotor signals and the postdictive influence of agency in the intentional binding paradigm, where participants always received an action confirmation with a visual, auditory or haptic outcome (in contrast to Coyle's work where only audio feedback was considered). Although we obtained different intentional binding values from the tasks involving gesture input and visual feedback in both studies, we found no statistically significant difference in this condition between Studies 1 and 2. 
Our results from both studies showed different outcome binding effects depending on the type of feedback, with audio and haptic feedback producing higher intentional binding effect than visual feedback. Visual feedback onscreen produced the lowest intentional binding effect in both studies. This suggests that participants perceived higher perception of controlling the touchless interface when they received an auditory or haptic confirmation, rather than a visual confirmation. In cognitive neuroscience, a wide range of studies have employed audio feedback for studying agency, showing it to be a suitable technique to measure and produce SoA [1, 27, 43, 44]. However, in our Study 2, we also found an intentional binding effect with vibrotactile and mid-air haptic outcomes with no statistically significant difference between them. This suggests that if one cannot provide audio feedback it may be preferred from an intentional binding perspective to provide haptic feedback over visual-only feedback.

It is worth mentioning that we are aware that the UltraHaptics device produces sound because of the ultrasound waves emission. In the frequency at which it works, audible sound is generated from its speakers. To address this, participants were asked to wear noisecancelling headphones, not only during this condition but also for the entire task (including all the conditions).

\section{Limitations}

For the present work, we only collected quantitative measures. We employed the intentional binding paradigm as an implicit measure of the SoA following evidence that suggests that the increased intentional binding is related to higher experience of agency [14, 45]. However, previous studies have suggested that self-reports of agency and intentional binding may operate differently [53], therefore further research is need to investigate the relation between explicit judgement of agency and intentional binding for touchless interfaces. Additionally, in this work we put more attention on the impact of output modalities on agency and further studies are needed to examine the effect of proprioceptive perception on the SoA in mid-air interactions, possibly by using the haptic devices to create more natural perception of touching real objects. We mainly compared visual feedback with the other modalities in our two studies, thereby more direct comparison between audio and haptic feedback will be explored in future work.

\section{Application scenarios}

In this work, we have shown types of interaction that significantly impact on users' SoA in order to provide solutions to improve touchless interfaces. Our results suggest that audio and haptic feedback are better to produce users' SoA compared with visual feedback. Although these kinds of feedback have been frequently used in past work (as seen in the related work section) the role of SoA have been unexplored. Here, we have validated these feedback types by implicit and quantitative metrics supporting their use to provide a better and more responsive interaction. Here we explain some possible application scenarios.

Interactions in Virtual Reality (VR) commonly rely on touchless actions; however, these systems often add haptic feedback, as they try to simulate real world settings in order to provide a realistic interaction. We have demonstrated that touch and touchless input modalities accompanied by mid-air haptic feedback improve users' intentional binding, which enables application scenarios for VR and bare-hands interactions. For example, by considering the role of agency in designing VR training simulators (e.g. flight or surgery), the designer can approximate agency effects in users that are similar to those in a real-life situation. In this way, their commitment to the interaction (action inputs and system responses) might be stronger, enabling better training for the professional.

It is known that audio and haptic feedback releases the visual channel to focus on additional tasks; this interplay is suitable for driving scenarios, for example. Our results showed that audio and mid-air haptic feedback improve users' SoA. This suggests that these kinds of feedback not only will help to focus driving attention but also produce the user's feeling of being in control during touchless interaction (e.g. controllers for car dashboards). Additionally, mid-air haptic feedback represents a good means for private communication in cases where audio cannot be played, allowing the user to still experience agency. By considering the SoA in interface design, we can explore a wide range of interaction paradigms that enable users' feeling of control in order create interactions that are more realistic and thus develop more responsive systems.

\section{CONCLUSION}

Despite touchless systems being used in a wide range of applications, the role of agency in these systems had been unexplored to date. The lack of understanding this aspect, constrains the relevance of perceiving a responsive interface. In this paper, we have demonstrated by implicit and quantitative measures that touchless input could be perceived as responsive as a physical input action. Although our work focused only on a basic activation gesture (in-air button press), this creates an opportunity to offer possible solutions for designers in order to improve gesture-based touchless interfaces. Our work also suggests that audio and haptic feedback in gesture-based touchless interactions are a good candidate for increasing users' sense of being in control and feeling of interacting with a more responsive system. These findings contribute to a new area for $\mathrm{HCI}$ researchers to explore agency consideration in $\mathrm{HCI}$ design.

\section{ACKNOWLEDGEMENTS}

We thank Luis Veloso for the video. This work has been supported by the European commission through European Research Council's starting grant (\#278576) and the Mexican National Council of Science and Technology. 


\section{REFERENCES}

1. Henk Aarts, Erik Bijleveld, Ruud Custers, Myrthel Dogge, Merel Deelder, Dennis Schutter, and Neeltje EM van Haren. 2012. Positive priming and intentional binding: Eye-blink rate predicts reward information effects on the sense of agency. Social Neuroscience 7 , 1: 105-112. DOI: http://dx.doi.org/10.1080/17470919.2011.590602

2. Steven Ashley. 2014. Touch-less control coming cars. SAE, automotive engineering: 20-23 from http://www.visteon.com/media/touchless.pdf

3. Domna Banakou and Mel Slater. 2014. Body ownership causes illusory self-attribution of speaking and influences subsequent real speaking. Proceedings of the National Academy of Sciences 111, 49: 1767817683. DOI: http://dx.doi.org/10.1073/pnas.1414936111

4. Laura Bartoli, Franca Garzotto, Mirko Gelsomini, Luigi Oliveto, and Matteo Valoriani. 2014. Designing and evaluating touchless playful interaction for ASD children. ACM, Proceedings of the 2014 conference on Interaction design and children: 17-26. DOI: http://dx.doi.org/10.1145/2593968.2593976

5. Thomas Baudel and Michel Beaudouin-Lafon. 1993. Charade: remote control of objects using free-hand gestures. Communications of the ACM 36, 7: 28-35. DOI: http://dx.doi.org/10.1145/159544.159562.

6. Sarah-Jayne Blakemore, Daniel M Wolpert, and Christopher D Frith. 2002. Abnormalities in the awareness of action. Trends in cognitive sciences 6, 6: 237-242. DOI: http://dx.doi.org/10.1016/S1364-6613(02)01907-1

7. Claude Cadoz. 1994. Les réalités virtuelles.

8. Tom Carter, Sue Ann Seah, Benjamin Long, Bruce Drinkwater, and Sriram Subramanian. 2013. UltraHaptics: multi-point mid-air haptic feedback for touch surfaces. ACM, Proceedings of the 26th annual ACM symposium on User interface software and technology: 505-514. DOI: http://dx.doi.org/10.1145/2501988.2502018

9. Valerian Chambon and Patrick Haggard. 2013. Premotor or Ideomotor: How Does the Experience of Action Come About? Action science: Foundations of an emerging discipline: 359 . DOI: http://dx.doi.org/10.7551/mitpress/9780262018555.003.001 4

10. Valerian Chambon and Patrick Haggard. 2012. Sense of control depends on fluency of action selection, not motor performance. Cognition 125, 3: 441-451. DOI: http://dx.doi.org/10.1016/j.cognition.2012.07.011

11. Jacob Cohen. 1977. Statistical power analysis for the behavioral sciences (revised ed.). New York: Academic Press.
12. David Coyle, James Moore, Per Ola Kristensson, Paul Fletcher, and Alan Blackwell. 2012. I did that! Measuring users' experience of agency in their own actions. ACM, Proceedings of the SIGCHI Conference on Human Factors in Computing Systems: 2025-2034. DOI: http://dx.doi.org/10.1145/2207676.2208350

13. Nicole David, Albert Newen, and Kai Vogeley. 2008. The "sense of agency" and its underlying cognitive and neural mechanisms. Consciousness and cognition 17, 2: 523-534. DOI: http://dx.doi.org/10.1016/j.concog.2008.03.004

14. Jeffrey P Ebert and Daniel M Wegner. 2010. Time warp: Authorship shapes the perceived timing of actions and events. Consciousness and cognition 19, 1: 481-489. DOI: http://dx.doi.org/10.1016/j.concog.2009.10.002

15. Kai Engbert, Andreas Wohlschläger, and Patrick Haggard. 2008. Who is causing what? The sense of agency is relational and efferent-triggered. Cognition 107, 2: 693-704. DOI: http://dx.doi.org/10.1016/j.cognition.2007.07.021

16. Euan Freeman, Stephen Brewster, and Vuokko Lantz. 2016. Do That, There: An Interaction Technique for Addressing In-Air Gesture Systems. ACM, Proceedings of the 2016 CHI Conference on Human Factors in Computing Systems. : 2319-2331. DOI: http://dx.doi.org/10.1145/2858036.2858308

17. Pablo Garaizar, Miguel A Vadillo, Diego López-deIpiña, and Helena Matute. 2014. Measuring software timing errors in the presentation of visual stimuli in cognitive neuroscience experiments. PloS one 9, 1: e85108. DOI: http://dx.doi.org/10.1371/journal.pone.0085108

18. Nicolas Georgieff and Marc Jeannerod. 1998. Beyond consciousness of external reality: a "who" system for consciousness of action and self-consciousness. Consciousness and cognition 7, 3: 465-477. DOI: http://dx.doi.org/10.1006/ccog.1998.0367

19. George A Gescheider, John H Wright, and Ronald T Verrillo. 2010. Information-processing channels in the tactile sensory system: A psychophysical and physiological analysis. Psychology Press: 136.

20. Ivan Golod, Felix Heidrich, Christian Möllering, and Martina Ziefle. 2013. Design principles of hand gesture interfaces for microinteractions. ACM, Proceedings of the 6th International Conference on Designing Pleasurable Products and Interfaces: 11-20. DOI: http://dx.doi.org/10.1145/2513506.2513508

21. Sidhant Gupta, Dan Morris, Shwetak N Patel, and Desney Tan. 2013. Airwave: Non-contact haptic feedback using air vortex rings. ACM, Proceedings of the 2013 ACM international joint conference on 
Pervasive and ubiquitous computing: 419-428. DOI: http://dx.doi.org/10.1145/2493432.2493463

22. Patrick Haggard and Manos Tsakiris. 2009. The experience of agency feelings, judgments, and responsibility. Current Directions in Psychological Science 18, 4: 242-246. DOI: https://doi.org/10.1111/j.1467-8721.2009.01644.x

23. Patrick Haggard, Sam Clark, and Jeri Kalogeras. 2002. Voluntary action and conscious awareness. Nature neuroscience 5, 4: 382-385. DOI: http://dx.doi.org/10.1038/nn827

24. Chris Harrison, Desney Tan, and Dan Morris. 2010. Skinput: appropriating the body as an input surface. ACM, Proceedings of the SIGCHI Conference on Human Factors in Computing Systems: 453-462. DOI: http://dx.doi.org/10.1145/1753326.1753394

25. Marc Jeannerod. 1999. The 25th Bartlett Lecture: To act or not to act: Perspectives on the representation of actions. The Quarterly Journal of Experimental Psychology A: Human Experimental Psychology 52, 1: 1-29 from http://www.tandfonline.com/doi/abs/10.1080/713755803

26. Petter Johansson, Lars Hall, Sverker Sikström, and Andreas Olsson. 2005. Failure to detect mismatches between intention and outcome in a simple decision task. Science 310, 5745: 116-119. DOI: http://dx.doi.org/10.1126/science.1111709

27. Nima Khalighinejad and Patrick Haggard. 2015. Modulating human sense of agency with non-invasive brain stimulation. Cortex 69: 93-103. DOI: http://dx.doi.org/10.1016/j.cortex.2015.04.015

28. Elena Kokkinara, Konstantina Kilteni, Kristopher J Blom, and Mel Slater. 2016. First Person Perspective of Seated Participants Over a Walking Virtual Body Leads to Illusory Agency Over the Walking. Scientific Reports 6. DOI: http://dx.doi.org/10.1038/srep28879

29. Thomas Kopinski, Stefan Geisler, and Uwe Handmann. 2013. Contactless Interaction for Automotive Applications. Mensch \& Computer Workshopband: $87-$ 94 from http://dl.mensch-undcomputer.de/handle/123456789/3368

30. Benjamin Libet, Curtis A Gleason, Elwood W Wright, and Dennis K Pearl. 1983. Time of conscious intention to act in relation to onset of cerebral activity (readiness-potential). Brain 106, 3: 623-642. DOI: https://doi.org/10.1093/brain/106.3.623

31. Jaime Lien, Nicholas Gillian, M Emre Karagozler, Patrick Amihood, Carsten Schwesig, Erik Olson, Hakim Raja, and Ivan Poupyrev. 2016. Soli: ubiquitous gesture sensing with millimeter wave radar. ACM Transactions on Graphics (TOG) 35, 4: 142. DOI: http://dx.doi.org/10.1145/2897824.2925953
32. Hannah Limerick, James W Moore, and David Coyle. 2015. Empirical evidence for a diminished sense of agency in speech interfaces. ACM, Proceedings of the 33rd Annual ACM Conference on Human Factors in Computing Systems: 3967-3970. DOI: http://dx.doi.org/10.1145/2702123.2702379

33. Mingyu Liu, Mathieu Nancel, and Daniel Vogel. 2015. Gunslinger: Subtle Arms-down Mid-air Interaction. ACM, Proceedings of the 28th Annual ACM Symposium on User Interface Software \& Technology: 63-71. DOI: http://dx.doi.org/10.1145/2807442.2807489

34. Takaki Maeda, Motoichiro Kato, Taro Muramatsu, Satoru Iwashita, Masaru Mimura, and Haruo Kashima. 2012. Aberrant sense of agency in patients with schizophrenia: Forward and backward over-attribution of temporal causality during intentional action. Psychiatry research 198, 1: 1-6. DOI: http://dx.doi.org/10.1016/j.psychres.2011.10.021

35. Yasutoshi Makino, Yoshikazu Furuyama, Seki Inoue, and Hiroyuki Shinoda. 2016. HaptoClone (HapticOptical Clone) for Mutual Tele-Environment by Realtime 3D Image Transfer with Midair Force Feedback. ACM, Proceedings of the 2016 CHI Conference on Human Factors in Computing Systems: 1980-1990. DOI: http://dx.doi.org/10.1145/2858036.2858481

36. Anders Markussen, Mikkel Rønne Jakobsen, and Kasper Hornbæk. 2014. Vulture: a mid-air wordgesture keyboard. ACM, Proceedings of the SIGCHI Conference on Human Factors in Computing Systems: 1073-1082. DOI: http://dx.doi.org/10.1145/2556288.2556964

37. John E McEneaney. 2013. Agency effects in humancomputer interaction. International Journal of HumanComputer Interaction 29, 12: 798-813. DOI: http://dx.doi.org/10.1080/10447318.2013.777826

38. Jess McIntosh, Charlie McNeill, Mike Fraser, Frederic Kerber, Markus Löchtefeld, and Antonio Krüger. 2016. EMPress: Practical Hand Gesture Classification with Wrist-Mounted EMG and Pressure Sensing. ACM, Proceedings of the 2016 CHI Conference on Human Factors in Computing Systems: 2332-2342. DOI: http://dx.doi.org/10.1145/2858036.2858093

39. Clive S Mellor. 1970. First rank symptoms of schizophrenia: I. The frequency in schizophrenics on admission to hospital. II. Differences between individual first rank symptoms. The British Journal of Psychiatry 117(536): 15-23 from http://bjp.rcpsych.org/content/117/536/15.fulltext.pdf+html

40. David Holz Michael Buckwald. 2010. Leap Motion, Inc. www.leapmotion.com

41. Yasuaki Monnai, Keisuke Hasegawa, Masahiro Fujiwara, Kazuma Yoshino, Seki Inoue, and Hiroyuki 
Shinoda. 2014. HaptoMime: mid-air haptic interaction with a floating virtual screen. ACM, Proceedings of the 27th annual ACM symposium on User interface software and technology: 663-667. DOI: http://dx.doi.org/10.1145/2642918.2647407

42. James Moore and Patrick Haggard. 2008. Awareness of action: Inference and prediction. Consciousness and cognition 17, 1: 136-144. DOI: http://dx.doi.org/10.1016/j.concog.2006.12.004

43. James W Moore and Sukhvinder S Obhi. 2012. Intentional binding and the sense of agency: a review. Consciousness and cognition 21, 1: 546-561. DOI: http://dx.doi.org/10.1016/j.concog.2011.12.002

44. James W Moore, Daniel M Wegner, and Patrick Haggard. 2009. Modulating the sense of agency with external cues. Consciousness and cognition 18, 4: 1056-1064. DOI: http://dx.doi.org/10.1016/j.concog.2009.05.004

45. James W Moore and Patrick Haggard. 2010. Intentional binding and higher order agency experience. Consciousness and cognition 19, 1: 490491. DOI: http://dx.doi.org/10.1016/j.concog.2009.11.007

46. James W Moore, Susanne A Schneider, Petra Schwingenschuh, Giovanna Moretto, Kailash P Bhatia, and Patrick Haggard. 2010. Dopaminergic medication boosts action-effect binding in Parkinson's disease. Neuropsychologia 48, 4: 1125-1132. DOI: http://dx.doi.org/10.1016/j.neuropsychologia.2009.12.014

47. Giovanna Moretto, Eamonn Walsh, and Patrick Haggard. 2011. Experience of agency and sense of responsibility. Consciousness and cognition 20, 4: 1847-1854. DOI: http://dx.doi.org/10.1016/j.concog.2011.08.014

48. Jörg Müller, Matthias Geier, Christina Dicke, and Sascha Spors. 2014. The boomRoom: mid-air direct interaction with virtual sound sources. ACM, Proceedings of the SIGCHI Conference on Human Factors in Computing Systems: 247-256. DOI: http://dx.doi.org/10.1145/2556288.2557000

49. Rajalakshmi Nandakumar, Vikram Iyer, Desney Tan, and Shyamnath Gollakota. 2016. FingerIO: Using Active Sonar for Fine-Grained Finger Tracking. ACM, Proceedings of the 2016 CHI Conference on Human Factors in Computing Systems: 1515-1525. DOI: http://dx.doi.org/10.1145/2858036.2858580

50. Rachel Nuwer. 2013. Armband adds a twitch to gesture control. New Scientist 217, 2906: 21. DOI: http://dx.doi.org/10.1016/S0262-4079(13)60542-4

51. Kenton O'Hara, Gerardo Gonzalez, Abigail Sellen, Graeme Penney, Andreas Varnavas, Helena Mentis, Antonio Criminisi, Robert Corish, Mark Rouncefield, and Neville Dastur. 2014. Touchless interaction in surgery. Communications of the ACM 57, 1: 70-77. DOI: http://dx.doi.org/10.1145/2541883.2541899

52. Sukhvinder Obhi and Patrick Haggard. 2004. Free Will and Free Won't Motor activity in the brain precedes our awareness of the intention to move, so how is it that we perceive control? American Scientist 92, 4: 358-365. DOI: http://dx.doi.org/10.1511/2004.4.358

53. Sukhvinder S Obhi and Preston Hall. 2011. Sense of agency and intentional binding in joint action. Experimental brain research 211, 3-4: 655-662. DOI: http://dx.doi.org/10.1007/s00221-011-2675-2

54. Sukhvinder S Obhi and Preston Hall. 2011. Sense of agency in joint action: Influence of human and computer co-actors. Experimental brain research 211, 3-4: 663-670. DOI: http://dx.doi.org/10.1007/s00221-011-2662-7

55. Dan Saffer. 2008. Designing gestural interfaces: Touchscreens and interactive devices. " O'Reilly Media, Inc.": 272.

56. Ben Shneiderman. 2005. Shneiderman's eight golden rules of interface design. Retrieved July 25: 2009 from https://www.cs.umd.edu/users/ben/goldenrules.html

57. Rajinder Sodhi, Ivan Poupyrev, Matthew Glisson, and Ali Israr. 2013. AIREAL: interactive tactile experiences in free air. ACM Transactions on Graphics (TOG) 32, 4: 134. DOI: http://dx.doi.org/10.1145/2461912.2462007

58. Neil Stewart. 2006. Millisecond accuracy video display using OpenGL under Linux. Behavior research methods 38, 1: 142-145. DOI: http://dx.doi.org/10.3758/BF03192759

59. Matthis Synofzik, Gottfried Vosgerau, and Martin Voss. 2013. The experience of agency: an interplay between prediction and postdiction. Frontiers in Psychology 4, 127. DOI: http://dx.doi.org/10.3389/fpsyg.2013.00127

60. Keisuke Takahata, Hidehiko Takahashi, Takaki Maeda, Satoshi Umeda, Tetsuya Suhara, Masaru Mimura, and Motoichiro Kato. 2012. It's not my fault: Postdictive modulation of intentional binding by monetary gains and losses. PloS one 7, 12: e53421. DOI: http://dx.doi.org/10.1371/journal.pone.0053421

61. Jonathan Taylor, Lucas Bordeaux, Thomas Cashman, Bob Corish, Cem Keskin, Toby Sharp, Eduardo Soto, David Sweeney, Julien Valentin, and Benjamin Luff. 2016. Efficient and precise interactive hand tracking through joint, continuous optimization of pose and correspondences. ACM Transactions on Graphics (TOG) 35, 4: 143. DOI: http://dx.doi.org/10.1145/2897824.2925965

62. Daniel M Wegner. 2003. The mind's best trick: how we experience conscious will. Trends in cognitive sciences 
7, 2: 65-69. DOI:

http://dx.doi.org/10.1016/S13646613(03)00002-0

63. Daniel M Wegner, Betsy Sparrow, and Lea Winerman. 2004. Vicarious agency: experiencing control over the movements of others. Journal of personality and social psychology 86, 6: 838. DOI:

http://dx.doi.org/10.1037/0022-3514.86.6.838

64. Dorit Wenke, Stephen M Fleming, and Patrick Haggard. 2010. Subliminal priming of actions influences sense of control over effects of action. Cognition 115, 1: 26-38. DOI:

http://dx.doi.org/10.1016/j.cognition.2009.10.016

65. Daniel Wigdor and Dennis Wixon. 2011. Brave NUI world: designing natural user interfaces for touch and gesture. Elsevier, 36. DOI:

http://dx.doi.org/10.1145/2047414.2047439
66. Wenjie Wu and Stefan Rank. 2015. Spatial Audio Feedback for Hand Gestures in Games. ACM, Proceedings of the 3rd ACM Symposium on Spatial User Interaction: 135-135. DOI: http://dx.doi.org/10.1145/2788940.2794363

67. Wenjie Wu and Stefan Rank. 2015. Responsive Environmental Diegetic Audio Feedback for Hand Gestures in Audio-Only Games. ACM, Proceedings of the 2015 Annual Symposium on Computer-Human Interaction in Play: 739-744. DOI: http://dx.doi.org/10.1145/2793107.2810336

68. Zhengyou Zhang. 2012. Microsoft kinect sensor and its effect. IEEE multimedia 19, 2: 4-10. DOI: http://dx.doi.org/10.1109/MMUL.2012.24 